\title{
Sequential administration of anti-VEGF therapy after craniospinal irradiation and intrathecal chemotherapy for the treatment of central nervous system AML relapse after allo-HSCT: a case report and literature review
}

\author{
Hanyu Cao ${ }^{1}$, Tao Tao ${ }^{2}$, Xiang-Dong Shen ${ }^{1}$, Lian Bai $^{1}$, Chao-Ling Wan ${ }^{1}$, De-Pei Wu ${ }^{1}$, \\ Jin-Li Li ${ }^{1}$, and Shengli Xue ${ }^{3}$ \\ ${ }^{1}$ First Affiliated Hospital of Soochow University \\ 2 the Fifth People's Hospital of Suzhou \\ ${ }^{3}$ The First Affilicated Hospital of Soochow University
}

December 23, 2021

\begin{abstract}
There have been few reports on the treatment of central nervous system (CNS) acute myeloid leukemia (AML) relapse. This case study demonstrates that bevacizumab may be a viable treatment option when combined with IT chemotherapy as maintenance therapy for those with CNS leukemia.
\end{abstract}

\section{Hosted file}

Sequential administration of anti-VEGF therapy after craniospinal irradiation and intrathecal chemother available at https://authorea.com/users/452458/articles/550531-sequential-administrationof-anti-vegf-therapy-after-craniospinal-irradiation-and-intrathecal-chemotherapy-forthe-treatment-of-central-nervous-system-aml-relapse-after-allo-hsct-a-case-report-andliterature-review 

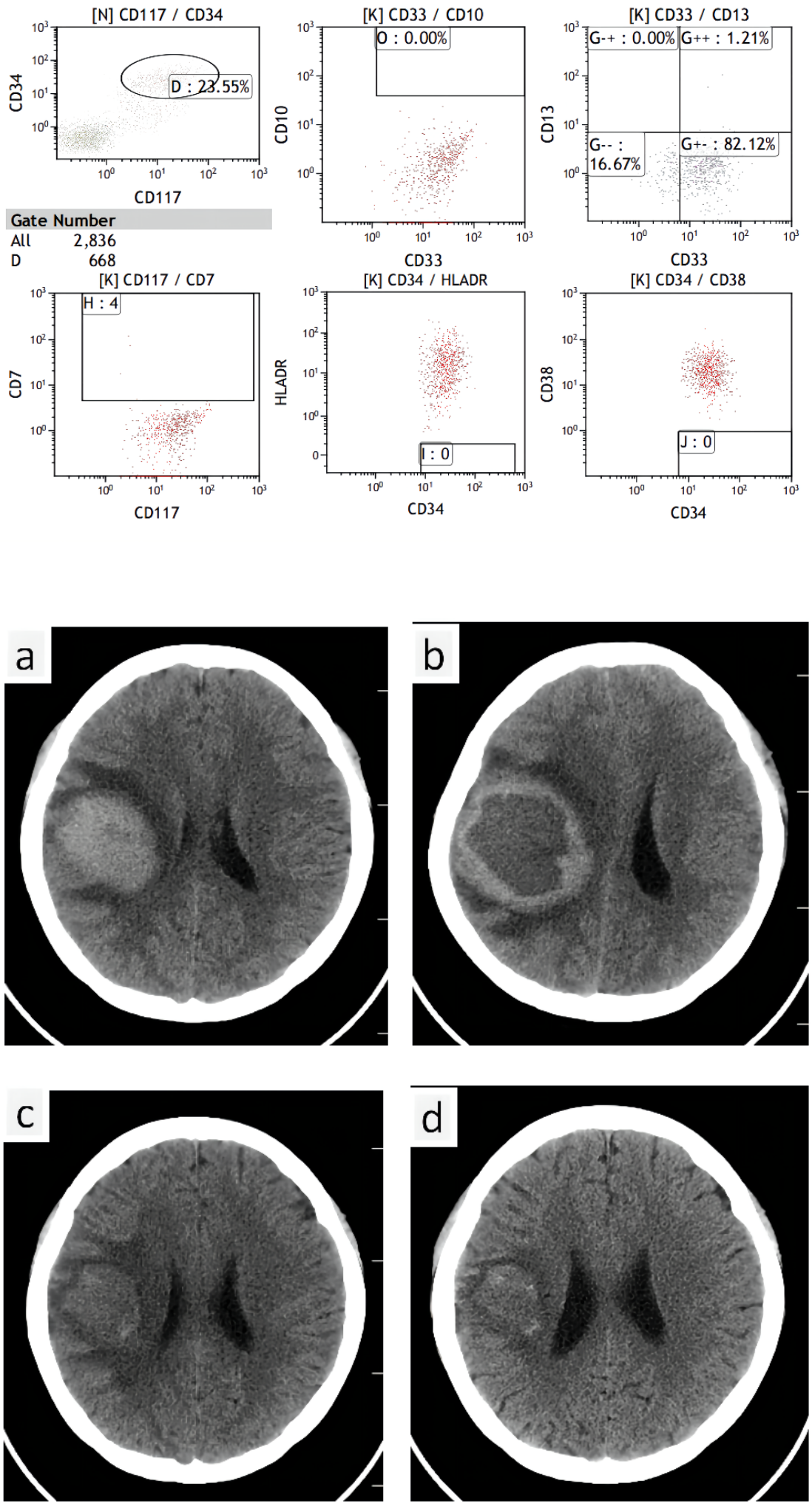

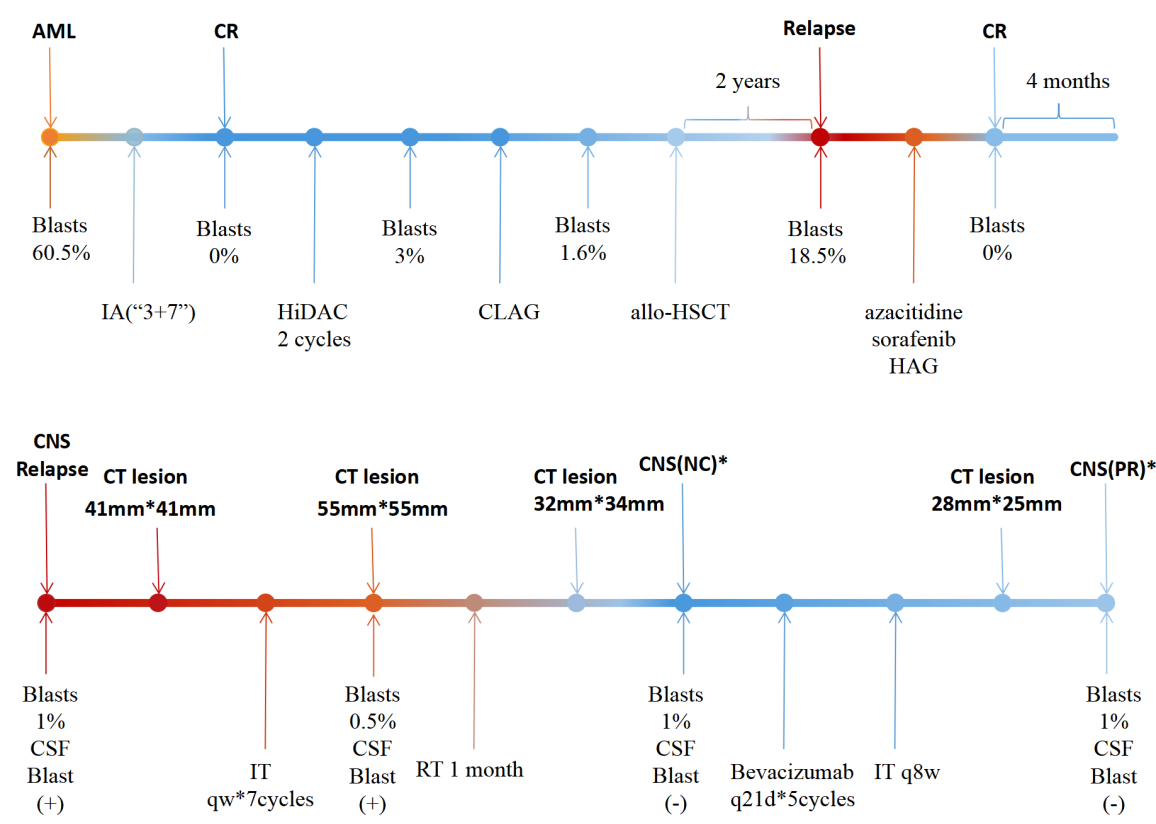